\title{
Die kognitiv-metaphorische Motiviertheit beim Erlernen von Idiomen am Beispiel eines Aufgabenblattes
}

\author{
Tamás Kispál (Szeged)
}

\begin{abstract}
The cognitive metaphorical motivation of idioms is one of the most treated subjects in English-language articles about teaching of idioms. In German-language phraseodidactic literature it is a rather rare phenomenon. The metaphorical motivation of several idiomatic expressions is undoubted. In this article we argue for their cognitive metaphorical motivation. It is hypothesized that the joint representation of several typical context examples, with idioms of the same conceptual metaphor, not only promotes the recognition and understanding of idioms but also the detection of cognitive metaphorical motivation of idioms. Context-sensitive tasks make a significant contribution to uncovering and learning the metaphorical idioms for learners of German as a foreign language. The learning-enhancing role of this method for the acquisition of idioms and their meanings are to be illustrated with the example of the conceptual metaphor LIFE IS A JOURNEY, the metaphorical idioms based on this conceptual metaphor, and an example worksheet.
\end{abstract}

\section{$1 \quad$ Einleitung und Problemaufriss}

Idiome werden traditionell als semantisch unanalysierbare feste Wortverbindungen angesehen, die wegen ihrer Unmotiviertheit als ganze Einheiten im mentalen Lexikon gespeichert und auch als solche gelernt werden müssen. Das Erlernen von Idiomen könnte dadurch den Lernern des Deutschen als Fremdsprache enorme Schwierigkeiten bereiten. Es können jedoch mehrere Motivationsfaktoren für die Idiome angegeben werden, und diese Motivationsfaktoren können auch das Erlernen der Idiome fördern. Einen dieser Motivationsfaktoren stellen konzeptuelle Metaphern, wie z. B. LEBEN IST EIN WEG dar. Im Rahmen der kognitiven Metapherntheorie hat bereits Lakoff betont, dass die Bedeutung von vielen Idiomen nicht arbiträr ist: "in a very large number of cases, the meanings of idioms are not arbitrary" (Lakoff 1987: 448).

Die Motiviertheit von Idiomen wird heute kaum bezweifelt. Umstritten ist jedoch, ob diese Motiviertheit durch konzeptuelle Metaphern oder durch andere Faktoren zustande kommt. Dobrovol'skij (2007) betont zum Beispiel die frame- und symbolbasierte Erklärung der Motiviertheit der Idiome. Er schlägt allerdings vor, bei jedem Idiom die Motivationsfaktoren einzeln zu untersuchen. Für das englische Idiom to be in cloud nine oder seine deutsche Entsprechung im siebten Himmel sein könnte man beispielsweise laut Dobrovol'skij (2007: 795) neben der konzeptuellen Metapher HAPPY IS UP u.a. die mit der Zahl SIEBEN verbundene symbolbasierte Motiviertheit angeben. Ebenfalls plädiert Kispál (2010a) für die mehrfache Motiviertheit der Idiome und die Methodenkombination bei ihrer metaphorischen Untersuchung am Beispiel der metaphorischen Idiome des Lebens. Im vorliegenden Beitrag wird die Motiviertheit der Idiome durch konzeptuelle Metaphern - u.a. im Hinblick auf einschlägige psycholinguistische Untersuchungen (z.B. Katz/Taylor 2008) - als ein 
möglicher Ansatz angenommen und der strukturierende Charakter der konzeptuellen Metaphern bei der Motiviertheit der metaphorischen Idiome als maßgeblich betrachtet.

Empirische Forschungen von Beréndi/Csábi/Kövecses (2008) haben nachgewiesen, dass die Bewusstmachung der konzeptuellen Metaphern zum Verstehen und Einprägen der figurativen Lexik beitragen kann: "an enhanced awareness of conceptual metaphors and metonymies on the part of language learners can help them comprehend and remember figurative lexis" (Beréndi/Csábi/Kövecses 2008: 65).

\section{$2 \quad$ Ziel der Untersuchung}

Die Motiviertheit der Idiome ist - wie oben erläutert - durch mehrere Faktoren bedingt. Im Folgenden werden die konzeptuellen Metaphern als eine der Motivationsgrundlagen der metaphorischen Idiome in den Vordergrund gestellt. Es wird von der Hypothese ausgegangen (cf. auch ibd.), dass die Aneignung der Grundlagen der kognitiven Metapherntheorie und entsprechende kontextsensitive Aufgaben einen wesentlichen Beitrag zum Aufdecken und Erlernen der metaphorischen Idiome bei Lernern des Deutschen als Fremdsprache leisten. Die lernfördernde Rolle dieser Methode für den Idiomerwerb bzw. seine Bedeutungserschließung wird am Beispiel der leicht nachvollziehbaren konzeptuellen Metapher LEBEN IST EIN WEG und der metaphorischen Idiome (z. B. sein Leben verläuft in geregelten Bahnen, auf die schiefe Bahn kommen, aus der Bahn werfen), denen diese konzeptuelle Metapher zugrunde liegt, sowie mit einem dazugehörigen Beispiel-Aufgabenblatt (cf. Anhang), veranschaulicht.

\section{Idiom- und Metapherndefinition}

Idiome werden im Folgenden in einer prototypischen Definition - im Sinne von Dobrovol'skij - verstanden, "als reproduzierbare Wortkomplexe, die den Kernbereich des phraseologischen Systems ausmachen, indem sie zusätzliche Irregularitätsmerkmale in verschiedenen Kombinationen und mit einer unterschiedlichen Intensität aufweisen" (Dobrovol'skij 1995: 48), z. B. Nonkompositionalität, Allomorphie zwischen der formalen und semantischen Struktur, Fixiertheit des Konstituentenbestandes, unikale Konstituenten, Opakheit. Der Status der Idiome als eine prototypische radiale Kategorie ist hier zu betonen. Es gibt bessere und schlechtere Vertreter der Kategorie Idiom. Die metaphorischen Idiome gehören zwar nicht zur Gruppe der prototypischen Idiome, d.h. zu den besseren Vertretern mit den meisten Irregularitätsmerkmalen (z. B. ins Gras beißen, nicht alle Tassen im Schrank haben), weil ihre Motiviertheit oder die Isomorphie zwischen ihrer formalen und semantischen Struktur der prototypischen Idiomkategorie widersprechen, aber durch ihre Mehrgliedrigkeit, Festigkeit und Frequenz können sie als Idiome gelten (z. B. seinen Kopf riskieren 'sein Leben oder seine Existenz riskieren'; sein Schäfchen im Trockenen haben 'sich seinen Vorteil gesichert haben').

Bei der Definition der Metapher soll ihr kognitiver und sprachlicher Status gleichzeitig berücksichtigt werden. Einerseits werden Metaphern aus kognitiver Sicht als konzeptuelle Metaphern betrachtet, die unser Denken strukturieren. Die Metapher ist eine Projektion von einem konkreten Ausgangsbereich auf einen abstrakten Zielbereich. Die konzeptuelle Metapher LEBEN IST EIN WEG wird beispielsweise als eine Projektion vom abstrakten Bereich LEBEN auf den konkreten Bereich WEG verstanden. Andererseits sind Metaphern aus sprachlicher Sicht als sprachliche Ausdrücke anzusehen, die als Realisierungsformen der konzeptuellen Metaphern gelten. Metaphorische Ausdrücke, die die konzeptuelle Metapher LEBEN IST EIN WEG sprachlich realisieren, sind z. B. (in seinem Leben) am Scheideweg stehen, ein Leben auf der Überholspur oder sein Leben verläuft in geregelten Bahnen. Ferner stellt die Metapher in einer kognitiven Auffassung keine Abweichung von der Norm dar, sondern sie ist ein alltagssprachliches Phänomen. 


\section{Die Motiviertheit der Idiome durch die konzeptuelle Metapher LEBEN IST EIN WEG}

Im Folgenden wird die kognitiv-metaphorische Motiviertheit des Lebens durch die idiomatische Realisierung der konzeptuellen Metapher LEBEN IST EIN WEG thematisiert, um ihre Auswahl als Thema für das behandelte Beispiel-Aufgabenblatt zu begründen. Es wird gezeigt, dass die metaphorische Strukturierung einen wesentlichen Beitrag zum Erlernen der Idiome dieser konzeptuellen Metapher leisten kann.

Das Leben ist ein häufiger Zielbereich bei vielen Metaphern. Eine der häufigsten metaphorischen Konzeptualisierungen des Lebens ist ihre Konzeptualisierung als Weg. ${ }^{1}$ Bei ihrer sprachlichen Realisierung sind neben den metaphorischen Idiomen viele Ein-WortMetaphern (z. B. Bahn, Gleis, Phase, Punkt, Spur, Station) und metaphorische Komposita (Lebenslauf, Lebensweg, Lebensziel, Mittelpunkt, Überholspur, Weggefährte, Wendepunkt) vertreten. Für den vorliegenden Beitrag sind nur die metaphorischen Idiome relevant, die jedoch in vielen Fällen die obigen metaphorischen Einzelwörter oder Komposita enthalten. In einer empirischen Untersuchung zur idiomatischen Realisierung von Lebens-Metaphern wurden 42 Lebens-Idiome zur Metapher LEBEN IST EIN WEG herausgestellt (Kispál 2010a).

\subsection{Strukturierung der Idiome durch Verkehrsmittel als Metaphernkomponenten}

Die Idiome zur Metapher LEBEN IST EIN WEG rufen verschiedene Aspekte des Bildschemas WEG hervor. Eine mögliche Strukturierung dieser Idiome erfolgt durch die Spezifizierung der Verkehrsmittel, die in diesen metaphorischen Idiomen als Komponenten vorhanden sind.

Mehrere Idiome enthalten das Bild eines Fußweges durch ihre Komponenten: Man kann seinen Weg machen oder gehen, die Wege von zwei Personen können sich kreuzen, man kann auch am Kreuzweg oder am Scheideweg in seinem Leben stehen, aber auch einen Punkt erreichen oder überschreiten, oder man muss den dornigen Weg gehen.

Neben dem Fußweg kann in einigen Fällen das Bild einer Auto-, Bahn- oder Schifffahrt evoziert werden. Eine Autofahrt kann bei den Idiomen ein Leben auf der Überholspur, eine Bahnfahrt bei den Idiomen aus dem Gleis werfen/kommen/bringen/springen/geraten und eine Schifffahrt bei den Idiomen Schiffbruch erleiden und zu neuen Ufern assoziiert werden (cf. Abbildung 1). ${ }^{2}$

\footnotetext{
${ }^{1}$ Als Ergebnis einer empirischen Untersuchung jüngeren Datums kommt Kispál (2010a: 73) zum Schluss: "The five conceptual metaphors that have the motivation basis for the most idioms of life in German are as follows: LIFE IS A JOURNEY, LIFE IS A CONTAINER, LIFE IS A GAME, LIFE IS FOOD, and LIFE IS A PRECIOUS POSSESSION".

2 Zur Spezifizierung der Verkehrsmittel bei der Auswahl der Metaphernkomponenten (im Englischen) cf. auch Boers (2003: 233): "although 'LIFE IS A JOURNEY' (e. g., 'We'll have to get round many obstacles to get married', 'The quest for love and happiness') is clearly based on the MOTION image schema, it can be 'enriched' by specifying the kind of vehicles involved, such as trains (e. g., 'It's about time you got back onto the right track'), ships (e. g., 'She's been drifting without a real purpose in life'), cars (e. g., 'He's in the fast lane to success'), and so on".
} 


\begin{tabular}{|l|l|l|l|}
\hline \multicolumn{1}{|c|}{ Fußweg } & Autofahrt & Bahnfahrt & \multicolumn{1}{c|}{ Schifffahrt } \\
\hline $\begin{array}{l}\text { seinen Weg } \\
\text { machen/gehen } \\
\text { die Wege haben } \\
\text { sich gekreuzt } \\
\text { am Kreuzweg/ } \\
\begin{array}{l}\text { Scheideweg stehen } \\
\text { Pfad der Tugend } \\
\text { einen Punkt } \\
\text { erreichen/an } \\
\text { einem Punkt } \\
\text { ankommen } \\
\text { den dornigen Weg } \\
\text { gehen müssen }\end{array}\end{array}$ & ein Leben aufder & $\begin{array}{l}\text { aus dem Gleis } \\
\text { werfen/kommen/ } \\
\text { bringen/springen/ } \\
\text { geraten }\end{array}$ & $\begin{array}{l}\text { Schiffbruch } \\
\text { erleiden } \\
\text { zu neuen Ufern }\end{array}$ \\
\hline
\end{tabular}

Abb. 1: Strukturierung der Idiome aufgrund der Spezifizierung von Verkehrsmitteln als Metaphernkomponenten

\subsection{Strukturierung der Idiome durch systematische Entsprechungen der Projizierung}

Konzeptuelle Metaphern beruhen auf systematischen Entsprechungen zwischen einem Ausgangsbereich und einem Zielbereich, als Ergebnis von Projizierungen. Eine weitere mögliche Strukturierung der Idiome zur Metapher LEBEN IST EIN WEG kann folglich sichtbar gemacht werden, indem die Aspekte des Ausgangsbereichs den Aspekten des Zielbereichs entsprechend analog zugeordnet und Idiome, die diese hervorheben, jeweils aufgeführt werden (cf. Abbildung 2).

\begin{tabular}{|l|l|l|l|}
\hline \multicolumn{4}{|c|}{ LEBEN IST EIN WEG } \\
\hline Zielbereich & Ausgangsbereich & $\begin{array}{l}\text { Metaphorisches } \\
\text { Idiom }\end{array}$ & Bedeutung des Idioms \\
\hline $\begin{array}{l}\text { Schwierigkeiten im } \\
\text { Leben }\end{array}$ & $\begin{array}{l}\text { Hindernisse auf dem } \\
\text { Weg }\end{array}$ & dorniger Weg & $\begin{array}{l}\text { 'Leben(sabschnitt) mit } \\
\text { viel Leid und } \\
\text { Hindernissen' }\end{array}$ \\
\hline Bindungen im Leben & Brücken im Verkehr & $\begin{array}{l}\text { alle Brücken hinter } \\
\text { sich abbrechen }\end{array}$ & $\begin{array}{l}\text { 'sich von allen } \\
\text { bisherigen Bindungen } \\
\text { lösen und ein neues } \\
\text { Leben beginnen' }\end{array}$ \\
\hline sicherer Lebensverlauf & $\begin{array}{l}\text { sichere Fahrtfläche } \\
\text { (Bahn, Gleis, Spur) }\end{array}$ & $\begin{array}{l}\text { aus der Bahn/dem } \\
\text { Gleis geraten/geworfen } \\
\text { werden }\end{array}$ & $\begin{array}{l}\text { 'aus der gewohnten und } \\
\text { sicheren Ordnung des } \\
\text { Lebens geraten' }\end{array}$ \\
\hline Probleme im Leben & $\begin{array}{l}\text { Abkommen von der } \\
\text { sicheren Fahrbahn }\end{array}$ & $\begin{array}{l}\text { auf die schiefe Bahn } \\
\text { kommen }\end{array}$ & $\begin{array}{l}\text { 'ein unmoralisches } \\
\text { Leben beginnen' }\end{array}$ \\
\hline $\begin{array}{l}\text { Entscheidungen im } \\
\text { Leben }\end{array}$ & $\begin{array}{l}\text { Kreuzwege, } \\
\text { Scheidewege }\end{array}$ & $\begin{array}{l}\text { 'vor einer wichtigen } \\
\text { Entscheidung im Leben } \\
\text { stehen' }\end{array}$ \\
\hline
\end{tabular}

Abb. 2: Strukturierung der Idiome aufgrund von Entsprechungen bei der konzeptuellen Metapher LEBEN IST EIN WEG

\section{$5 \quad$ Arbeitsschritte zur Vorbereitung des Aufgabenblattes}

Die lernfördernde Rolle der im Abschnitt 2 erwähnten Methode, die darin besteht, kontextsensitive Aufgaben zum Erkennen der kognitiv-metaphorischen Motiviertheit von 
Idiomen $\mathrm{zu}$ erstellen, wird am Beispiel eines Aufgabenblattes veranschaulicht. Zur Anwendung dieser Methode und zur Vorbereitung dieses Aufgabenblattes werden folgende Arbeitsschritte vorgeschlagen:

\section{Einen metaphorischen Zielbereich auswählen}

Bei der Auswahl des Zielbereichs empfiehlt sich die Überlegung, welche Bereiche von den Fremdsprachenlernern mit konkreten alltagssprachlichen Ausdrücken (von den möglichen Ausgangsbereichen) belegt werden können. Geeignet sind z. B. Emotionen, wie ANGST, ÄRGER, FREUDE, GLÜCK, LIEBE, die aus der Perspektive der Idiome ziemlich produktiv und auch in kontrastiver Sicht vorteilhaft sind, weil die entsprechenden metaphorischen Konzepte als Grundlagen für gemeinsame kulturelle Modelle dienen können.

\section{Eine typische konzeptuelle Metapher des Zielbereichs festlegen}

Es ist sinnvoll, als Ausgangsbereich (Teil der konzeptuellen Metapher) ein von den Lernern offensichtlich leicht nachvollziehbares Gebiet der Alltagssprache auszuwählen. Dies sollte möglichst zum Grundwortschatz der jeweiligen Lernergruppe gehören. Die Auswahl eines gut strukturierten metaphorischen Bereichs wird empfohlen.

\section{Durch diese konzeptuelle Metapher motivierte Idiome sammeln}

Bei der Suche nach entsprechenden Idiomen können Wörterbücher oder Korpora zu Hilfe genommen werden. Im Gegensatz zu den häufig auf Introspektion beruhenden deutschsprachigen Wörterbüchern sollten (als Ergänzung) möglichst Korpora als Datenbasis für die Idiomform und die Idiombelege auf dem Aufgabenblatt verwendet werden.

\section{Authentische Texte finden, die diese Idiome enthalten}

Die Korpora im IDS-Korpusrecherche- und -analysesystem COSMAS II ${ }^{3}$ bieten zahlreiche alltagssprachliche Belege, vor allem aus der Pressesprache. Sie eignen sich auch für die Suche nach Belegen für metaphorische Idiome. ${ }^{4}$

Das Beispiel-Aufgabenblatt wurde anhand dieser Schritte folgendermaßen angefertigt:

1. Einen metaphorischen Zielbereich auswählen: Zum Zielbereich wurde das LEBEN, das bei vielen Metaphern vorkommt, ausgewählt.

2. Eine typische konzeptuelle Metapher festlegen: LEBEN IST EIN WEG ist eine typische und auch häufige konzeptuelle Metapher zum Zielbereich LEBEN. Davon zeugen viele Untersuchungen, die die Lebens-Metaphern einer Analyse unterziehen (Jäkel 2003: 261ff., Katz/Taylor 2008, Kövecses 2005: 184ff.). Der Ausgangsbereich WEG und viele damit zusammenhängende Ausdrücke aus dem Wortfeld Weg können zum Grundwortschatz gerechnet werden. 5

3. Durch diese konzeptuelle Metapher motivierte Idiome sammeln: Zur konzeptuellen Metapher LEBEN IST EIN WEG wurden folgende drei idiomatische Realisierungsformen für vorliegendes Aufgabenblatt ausgesucht: sein Leben verläuft in geregelten Bahnen, auf die schiefe Bahn kommen, aus der Bahn werfen. Die gemeinsame Komponente Bahn kann die Assoziation zur Reise und zum Weg seitens der Lerner erleichtern. ${ }^{6}$ Die Idiome auf dem

\footnotetext{
3 Im Internet unter www.ids-mannheim.de/cosmas2, Stand 15. Februar 2011.

${ }^{4}$ Auch andere Internetquellen kann man für Korpusbelege und damit für kognitiv-metaphorische Aufgaben mit Idiomen gut benutzen, obwohl meistens mit einer kleineren Beleganzahl, z.B. das Digitale Wörterbuch (www.dwds.de, Stand 15. Februar 2011), Online-Zeitschriftenarchive usw.

${ }^{5} \mathrm{Cf}$. http://wortschatz.uni-leipzig.de/, Stand 15. Februar 2011.

6 Acht von dreizehn Bedeutungen des Wortes Bahn in LGwDaF enthalten das Wort Weg oder Wörter aus dem Wortfeld der Reise. Neben Übereinstimmungen gibt es also auch Abweichungen in der Bedeutung der Wörter Bahn und Weg (cf. auch Zinken 2007: 453f.). Wegen ihrer gemeinsamen Behandlung bei den Weg-Metaphern
} 
Aufgabenblatt stammen aus einem Korpus, das als Ergebnis einer mehrdimensionalen Untersuchung entstand (cf. Kispál 2010a).

4. Authentische Texte finden, die diese Idiome enthalten: $\mathrm{Zu}$ jedem Idiom wurde ein Kontext aus den IDS-Korpora gesucht. Die Texte auf dem Aufgabenblatt stammen aus drei verschiedenen Tageszeitungen.

\section{Aufgabenblatt zum Erkennen der metaphorischen Motiviertheit von Idiomen}

Das Erlernen der Idiome kann durch das Erkennen ihrer metaphorischen Motiviertheit gefördert werden (cf. Beréndi/Csábi/Kövecses 2008). Im Folgenden wird ein BeispielAufgabenblatt vorgestellt, das zum Ziel hat, den Lerner des Deutschen als Fremdsprache auf die kognitiv-metaphorische Motiviertheit der Idiome aufmerksam zu machen. Die Aufgaben enthalten idiomatische Realisierungsformen der oben dargestellten konzeptuellen Metapher LEBEN IST EIN WEG. Das Lösen der Aufgaben auf diesem Aufgabenblatt besteht aus drei Phasen:

Phase 1: Erkennen von Idiomen (derselben konzeptuellen Metapher) in einigen authentischen Textausschnitten;

Phase 2: Verstehen von Idiomen im Kontext;

Phase 3: Erkennen der kognitiv-metaphorischen Motiviertheit von Idiomen.

Es wird von folgender These ausgegangen, die auf Erfahrungen des Autors des vorliegenden Aufsatzes beruht:

Das gemeinsame Aufführen von mehreren typischen Kontextbelegen, mit Idiomen derselben konzeptuellen Metapher, fördert nicht nur das Erkennen und das Verstehen der Idiome, sondern auch das Erkennen der kognitiv-metaphorischen Motiviertheit der Idiome.

\subsection{Erkennen von Idiomen im Kontext}

In der Phraseodidaktik wird von einem phraseologischen Dreischritt (Erkennen, Verstehen, Verwenden) (cf. Kühn 1992) bzw. in einer erweiterten Fassung von einem phraseologischen Vierschritt (Erkennen, Verstehen, Festigen, Verwenden) bei der Aneignung der Phraseologismen ausgegangen (cf. Lüger 1997: 101f.). Für das Üben der einzelnen Phasen sind verschiedene Aufgabentypen geeignet. Zum Erkennen der Idiome dienen am besten kontextspezifische Aufgaben. Der Text soll dabei die zentrale Bezugsgröße sein (Lüger 2004: 129).

Das Beispiel-Aufgabenblatt enthält drei kurze Textausschnitte mit jeweils einem Idiom zur konzeptuellen Metapher LEBEN IST EIN WEG: sein Leben verläuft in geregelten Bahnen, auf die schiefe Bahn kommen, aus der Bahn werfen.

(1) Bereits seit sechs Jahren wohnt Erich (43 Jahre) im Haus der jungen Arbeiter. Ihm gefällt es hier, denn sein Leben verläuft durch das Arbeitsprojekt und die Hilfe von Kaplan Bonetti in geregelten Bahnen. Weihnachten im "Haus" ist für ihn schon zur Routine geworden, und auf den Gottesdienst, das große Fest in der Gemeinschaft und das Geschenkpäckchen freut er sich schon - so wie die anderen auch. (Vorarlberger Nachrichten, 10.12.1998: Feiern und singen um nicht zu weinen)

(2) Zwanzig Monate hat der italienische Staatsbürger bereits abgesessen. Der Schreinergeselle kam, wie die Hauptverhandlung am Mittwoch ergab, schon in jugendlichem Alter auf die

sowie aus einem didaktischen Grund wird hier jedoch auf die Abweichungen nicht näher eingegangen. Außerdem sollte die Möglichkeit der metaphorischen Motiviertheit des Wortes Bahn in ihrer sport(fach)sprachenbezogenen Bedeutung bei den erwähnten Idiomen sein Leben verläuft in geregelten Bahnen, auf die schiefe Bahn kommen, aus der Bahn werfen offen bleiben. 
schiefe Bahn. Seinen Lebensunterhalt bestritt er in der Folge mit einer Reihe von Eigentumsdelikten. 1994 kam er in Untersuchungshaft, setzte seine kriminelle Karriere aber schon unmittelbar nach der Entlassung fort. Aus dieser Zeit wurden ihm jetzt von Staatsanwalt Joachim Böhn 25 Einbrüche und Autodiebstähle zur Last gelegt. (Frankfurter Rundschau, 07.03.1997: Fast fünf Jahre Haft wegen Einbruchsserie)

(3) Im Augenblick möchte Mattie eigentlich ihrem Vater für alles die Schuld geben. Sie ist wütend, weil er sie verlassen und ihr Leben dermaßen aus der Bahn geworfen hat. (Berliner Morgenpost, 06.11.1998)

Zur Lösung der ersten Aufgabe, dem Erkennen von Phraseologismen können verschiedene phraseologische Merkmale (phonetische, stilistische, strukturelle Abweichungen) beitragen, die dem Fremdsprachenlerner auffallen, ohne diese Merkmale sich davor angeeignet zu haben. Bei den Idiomen ist jedoch die semantische Abweichung, d.h. der Kontrast zwischen der wörtlichen und der idiomatischen Lesart am ehesten auffällig. Dies wird zweifelsohne im Kontext zunehmend ersichtlich, indem der Kontext die Idiome disambiguiert.

Bei der Disambiguierung kann die Metaphern-Identifizierungstheorie (MIP) helfen. Bei der von der Pragglejaz Group (2007) herausgearbeiteten MIP (metaphor identification procedure) handelt es sich um eine Analyse, bei der der Kontext eine wesentliche Rolle spielt. Vereinfacht kann das Wesen dieser Methode folgendermaßen beschrieben werden: Wenn die Bedeutung der einzelnen lexikalischen Einheiten im untersuchten Kontext zu der gegenwärtigen Basisbedeutung (anders ausgedrückt: der ursprünglichen Bedeutung oder der wörtlichen Bedeutung) dieser Lexeme im Gegensatz steht, aber im Kontrast dazu verstanden werden kann, handelt es sich um eine metaphorische lexikalische Einheit. Die letzten Schritte dieser deduktiven Analyse lauten in der Theoriebeschreibung folgendermaßen:

If the lexical unit has a more basic current-contemporary meaning in other contexts than the given context, decide whether the contextual meaning contrasts with the basic meaning but can be understood in comparison with it (...). If yes, mark the lexical unit as metaphorical. (Pragglejaz Group 2007: 3)

Wie auch von der Pragglejaz Group (ibd.: 25), wird auch hier die Konsultierung von äußeren Quellen, z. B. Wörterbüchern, empfohlen. Im Kontext von metaphorischen Ausdrücken können weitere lexikalische Elemente aus dem Wortschatz des Zielbereichs enthalten sein, die auch dem Lerner beim Erkennen und Verstehen von metaphorischen Idiomen im Kontext hilft. ${ }^{7}$ Ebenfalls helfen dabei diejenigen lexikalischen Einheiten im Kontext, die mit der Bedeutung der Idiome semantisch zusammenhängen.

In diesem Sinne erfolgt die Analyse des Erkennens der metaphorischen Idiome in den Texten des Beispiel-Aufgabenblattes: Die scheinbare semantische Unvereinbarkeit des abstrakten Ausdrucks Leben mit dem konkreten Wort Bahn fällt bereits bei der Nennform des Idioms sein Leben verläuft in geregelten Bahnen auf. Dies wird im Text 1 durch den Kontext verstärkt, in dem nichts auf die wörtliche Bedeutung des Wortes Bahn hinweist (z. B. 'ein Weg, den man sich macht, wenn Hindernisse da sind' (LGwDaF, Bedeutung 1)). Ebenfalls stellt das Leben im dritten Text mit der Komponente Bahn im Idiom aus der Bahn werfen eine semantische Unverträglichkeit dar: Der Vater von Mattie hat "ihr Leben dermaßen aus der Bahn geworfen". In der wörtlichen Lesart könnten bei dieser Wortverbindung z. B. folgende Bedeutungen von Bahn gemeint sein: 'eine abgegrenzte Strecke mit einer bestimmten Breite und Länge, auf der sportliche Wettkämpfe (Wettrennen o. ̈̈.) stattfinden' (LGwDaF, Bedeutung 4), oder 'Fahrspur' (LGwDaF, Bedeutung 6). Im zweiten Beispiel könnte das Subjekt (der Schreinergeselle) theoretisch nicht gegen die Selektionsbeschränkung des Verbs kommen im Idiom auf die schiefe Bahn kommen verstoßen, wenn eine wörtliche Bedeutung

7 Auch für die Extraktion von Metaphern aus Korpora schlägt Stefanowitsch (2006: 1ff.) bei zielbereichbezogenen Untersuchungen u.a. die Methode der Suche nach dem Wortschatz des Zielbereichs vor. 
(z. B. Bedeutung 4 aus dem LGWDaF, siehe oben) im Text gemeint wäre. Die wörtliche Lesart wäre hier allerdings seltsam, und der Kontext kann diese theoretisch mögliche Lesart nicht unterstützen.

\subsection{Verstehen von Idiomen im Kontext}

Der jeweilige Kontext hilft folgendermaßen beim Verstehen der Idiome. Im Text 1 könnten bereits zwei Komponenten des Idioms, geregelt und Leben ausreichen, um die Bedeutung des Idioms mit dem Leben zu verbinden ('ein ordentliches, geregeltes Leben führen'). Die Bedeutung eines geregelten Lebens wird aber auch noch im Kontext durch die Wörter Routine, gefällt, freut belegt. Folgen des geregelten Lebens von Erich (im Text) sind für ihn Routine, Zufriedenheit und Freude. Die Ausdrücke Untersuchungshaft, kriminelle Karriere, Einbrüche, Autodiebstähle unterstützen auch im Kontext des Idioms auf die schiefe Bahn kommen im Text 2 eindeutig seine Bedeutung 'ein unmoralisches, kriminelles Leben beginnen'. Ebenfalls können die lexikalischen Einheiten im Text 3 dem Leser helfen, einzusehen, dass die Situation, dass Matties Vater sie verlassen hat, und deshalb Mattie auf ihn wütend ist und ihm für alles die Schuld geben möchte, ein Zeichen dafür sein kann, dass ihr Leben 'die gewohnte Ordnung und Regelmäßigkeit verloren' hat, was durch das Idiom aus der Bahn werfen ausgedrückt wird.

Beim Verstehen von Idiomen im DaF-Unterricht können auch muttersprachliche bzw. sprachenpaarbezogene Aspekte berücksichtigt werden, was in einer homogenen Lernergruppe wesentlich einfacher als in einer multikulturellen Gruppe ist. Die Multikulturalität ist andererseits ein Vorteil für die Veranschaulichung des universellen Charakters der konzeptuellen Metaphern.

\subsection{Erkennen der kognitiv-metaphorischen Motiviertheit von Idiomen}

Im dritten Schritt soll auf die Verbindung zwischen den Idiomen auf dem Aufgabenblatt hingewiesen werden. Dies kann beispielsweise in Form einer einfachen Frage formuliert werden, die sich auf die Gemeinsamkeiten bei den Idiomen der aufgeführten Textausschnitte bezieht: Welche inhaltlichen Gemeinsamkeiten können Sie bei den Redewendungen dieser Textausschnitte entdecken?

Folgende Schritte fördern das Erkennen der kognitiv-metaphorischen Motiviertheit von Idiomen in einem induktiven Verfahren:

1. Erkennen der gemeinsamen Komponente in den aufgefundenen Idiomen: Auf dem Beispiel-Aufgabenblatt kann man eine formale Gemeinsamkeit zwischen den Idiomen erkennen. Alle Idiome enthalten die gemeinsame Komponente Bahn. Es handelt sich hier jedoch nicht um eine bloße Auflistung von Idiomen mit einer gemeinsamen Konstituente. Als Einstieg kann allerdings dieses Verfahren, in dem alle behandelten Idiome eine gemeinsame Komponente enthalten, zum leichteren Erkennen der metaphorischen Motiviertheit beitragen.

2. Erkennen des Zusammenhangs zwischen der wörtlichen und der metaphorischen Bedeutung der gemeinsamen Komponente im Kontext: Der Lerner kann erkennen, dass alle aufgeführten Idiome in den Textausschnitten das Leben mit den Komponenten der Bahnfahrt beschreiben, was auf dem Beispiel-Aufgabenblatt relativ leicht nachvollziehbar ist.

3. Erkennen der metaphorischen Analogie: Der Lerner kann auf die Analogie kommen: die geregelten Bahnen entsprechen Ordnung, Frieden, Normalität im Leben einer Person; Geradlinigkeit entspricht dem moralischen Leben, während Schiefe (der Bahn) mit dem unmoralischen Leben in Analogie steht (vgl. Abbildung 2).

4. Erkennen der konzeptuellen Metapher: Durch obige metaphorische Analogie kann schließlich die konzeptuelle Metapher LEBEN IST EIN WEG erkannt werden. Dieser letzte 
Schritt kann noch ggf. durch eine weitere Aufgabe zur Erweiterung der metaphorischen Idiome (z. B. durch Idiome mit Komponenten vom Fußweg oder von der Schifffahrt) (cf. Abbildung 1) gefördert werden.

Die Methode wurde auch in der universitären Lehre an der Universität Szeged erprobt und hat die lernerfördernde Rolle der konzeptuellen Metaphern nachgewiesen und selbst die Lernermotivation verstärkt. Ähnlich dem vorgestellten Arbeitsblatt wurden weitere Arbeitsblätter $\mathrm{zu}$ weiteren Lebens-Idiomen erstellt. Auf einem weiteren Aufgabenblatt standen die Idiome die Hauptrolle spielen, eine Hürde nehmen, Kopf und Kragen aufs Spiel setzen, die die metaphorischen Ausgangsbereiche SPORT und SPIEL belegen, und in den aufgeführten Kontexten auf das Leben hingewiesen haben. Ebenfalls konnte der Zielbereich LEBEN durch die Idiome ein Schattendasein führen, auf der Sonnenseite des Lebens stehen (bzw. den Ausgangsbereich LichT bzw. Leben als ZYKLUS) mithilfe von Kontextbelegen auf einem Aufgabenblatt interpretiert werden. ${ }^{8}$ Das Erkennen der kognitiv-metaphorischen Motiviertheit kann besonders in den Fällen eine zusätzliche hilfreiche und fruchtbare Methode im Fremdsprachenunterricht sein, wo die behandelten ausgangssprachlichen Idiome entweder keine oder nur partielle Äquivalente in der Zielsprache haben. ${ }^{9}$

Das primäre Ziel dieses Ansatzes ist es, die metaphorische Motivation zu veranschaulichen und als ein nächstes Ziel die metaphorische Kompetenz zu üben. Bei phraseodidaktischen Aufgaben zum Erkennen der kognitiv-metaphorischen Motiviertheit ist es sinnvoll, Idiome auszuwählen, die Komponenten aus der Alltagssprache enthalten und zum allgemeinen Grundwortschatz gehören (cf. Hallsteinsdóttir/Šajanková/Quasthoff 2006 zum phraseologischen Optimum für DaF). Die Auswahl der Idiome für kognitiv-metaphorische Aufgaben ist jedoch nicht alleine durch Kriterien wie Frequenz oder Geläufigkeit der behandelten Idiome bedingt, denn wichtig ist, dass sie $\mathrm{zu}$ einem typischen konkreten metaphorischen Ausgangsbereich gehören und damit zur Veranschaulichung der kognitivmetaphorischen Motiviertheit dieser lexikalischen Einheiten in den Aufgaben geeignet sind. Der korpusbasierte Ansatz sorgt zusätzlich für die Authentizität der behandelten Idiome.

\section{$7 \quad$ Zusammenfassung und Ausblick}

Das Ziel der Darstellung des Beispiel-Aufgabenblattes ist, eine Methode vorzustellen, mit der Lerner des Deutschen als Fremdsprache auf die kognitiv-metaphorische Motiviertheit der Idiome aufmerksam gemacht werden können. Die Methode basiert auf dem aus der Phraseodidaktik bekannten phasenbezogenen Phraseologieerwerb sowie auf einer kontextsensitiven Aufgabendarstellung. Durch die gemeinsame Aufführung von mehreren typischen Kontextbelegen auf einem Aufgabenblatt kann - neben Erkennen und Verstehen der Idiome - das Erkennen der kognitiv-metaphorischen Motiviertheit nicht nur der Idiome, sondern auch anderer Wortschatzeinheiten der gleichen konzeptuellen Metapher gefördert werden.

Im Weiteren können Lerner aufgefordert werden, nach der idiomatischen Realisierung von anderen konzeptuellen Metaphern, z. B. weiteren Ausgangsbereichen zum Zielbereich Leben (Behälter, Kampf, Spiel) zu suchen. Typische metaphorische Zielbereiche können weiterhin die Bereiche von Emotionen sein (z. B. Ärger, Glück, Liebe), die als Grundlagen für universelle metaphorische Konzepte zahlreiche Idiome enthalten. Dabei sollte immer die kontextsensitive Herangehensweise betont werden. Die eigene Verwendung von Korpora

\footnotetext{
${ }^{8}$ Zur Konzeptualisierung des menschlichen Lebens als Zyklus in deutschen Idiomen cf. Kispál (2010b).

${ }^{9}$ Die deutschen metaphorischen Idiome auf dem Beispiel-Aufgabenblatt haben z.B. im Ungarischen nur partielle idiomatische Äquivalente: sein Leben verläuft in geregelten Bahnen - élete sinen van/visszazökken a rendes kerékvágásba; auf die schiefe Bahn kommen - lejtöre kerül; aus der Bahn werfen - kizökkent a megszokott kerékvágásból.
} 
(möglich teilweise auch durch autonomes Lernen) kann den Entdeckungsprozess fördern und zur weiteren Lernermotivation beitragen (cf. MacArthur/Littlemore 2008). Die dargestellte Methode kann einen wesentlichen Beitrag zur Entwicklung der idiomatischen und der metaphorischen Kompetenz der Fremdsprachenlerner leisten. Es kann überlegt werden, inwieweit die konzeptuellen Metaphern den Fremdsprachenlernern auf weiteren Ebenen (z. B. mit der Thematisierung der konzeptuellen Metaphern vor oder nach dem Lösen des vorgestellten Aufgabenblattes) bewusst gemacht werden können (cf. Beréndi/Csábi/Kövecses 2008). Ein großer Vorteil dieser Methode besteht darin, dass Idiome nicht als isolierte Phänomene behandelt, sondern in die Arbeit mit und an Texten eingebunden werden. Außerdem wird die sprachlich-konzeptuelle Strukturierung der Welt mit einbezogen und mit der eigenen Welt konfrontiert. Die Grenzen des Aufgabenblattes, die v. a. in dem einseitigen Fokus auf die metaphorische Motiviertheit liegen, können durch das Einbeziehen anderer Mittel der Phraseodidaktik (dazu liefert dieses Themenheft viele Beispiele) und eine Erweiterung um andere Bereiche der Wortschatz- und Grammatikarbeit aufgebrochen werden.

\section{Literatur}

Beréndi, Márta/Csábi, Szilvia/Kövecses, Zoltán (2008): "Using conceptual metaphors and metonymies in vocabulary teaching". In: Boers, Frank/Lindstromberg, Seth (eds.): Cognitive Linguistic Approaches to Teaching Vocabulary and Phraseology. Berlin, de Gruyter: 65-99. (= Applications of Cognitive Linguistics 6).

Boers, Frank (2003): "Applied Linguistics Perspectives on Cross-Cultural Variation in Conceptual Metaphor". Metaphor and Symbol, 18.4: 231-238.

Dobrovol'skij, Dmitrij (1995): Kognitive Aspekte der Idiom-Semantik. Studien zum Thesaurus deutscher Idiome. Tübingen: Narr (= Eurogermanistik 8).

Dobrovol'skij, Dmitrij (2007): "Cognitive approaches to idiom analysis". In: Burger, Harald/Dobrovol'skij, Dmitrij/Kühn, Peter/Norrick, Neal R. (eds.): Phraseologie/ Phraseology. Ein internationales Handbuch zeitgenössischer Forschung/ An International Handbook of Contemporary Research. 2. Halbband. Berlin, de Gruyter: 789-818. (= Handbücher zur Sprach- und Kommunikationswissenschaft 28.2).

Hallsteinsdóttir, Erla/Šajánková, Monika/Quasthoff, Uwe (2006): "Phraseologisches Optimum für Deutsch als Fremdsprache. Ein Vorschlag auf der Basis von Frequenz- und Geläufigkeitsuntersuchungen". Linguistik Online 27: 117-136.

Jäkel, Olaf (2003): Wie Metaphern Wissen schaffen. Die kognitive Metapherntheorie und ihre Anwendung in Modell-Analysen der Diskursbereiche Geistestätigkeit, Wirtschaft, Wissenschaft und Religion. Hamburg: Dr. Kovac.

Katz, Albert N./Taylor, Tamsen E. (2008): "The Journeys of Life: Examining a Conceptual Metaphor with Semantic and Episodic Memory Recall". Metaphor and Symbol 23.3: 148173.

Kispál, Tamás (2010a): "Introspective data and corpus data. Combination instead of confrontation in the study of German metaphorical idioms of life". Sprachtheorie und germanistische Linguistik 20.1: 57-78.

Kispál, Tamás (2010b): "Frühling und Herbst, Sonne und Schatten. Die metaphorische Konzeptualisierung des menschlichen Lebens als Zyklus in deutschen Idiomen". In: Korhonen, Jarmo/Mieder, Wolfgang/Piirainen, Elisabeth/Pinel, Rosa (Hrsg.): Europhras 2008. Beiträge zur internationalen Phraseologiekonferenz vom 13.-16.8.2008 in Helsinki. Helsinki: Universität Helsinki. 100-112.

Kövecses, Zoltán (2005): Metaphor in Culture. Universality and Variation. Cambridge: Cambridge University Press. 
Kühn, Peter (1992): "Phraseodidaktik. Entwicklungen, Probleme und Überlegungen für den Muttersprachenunterricht und den Unterricht DaF". Fremdsprachen Lehren und Lernen 21: 169-189.

Lakoff, George (1987): Women, Fire and Dangerous Things. What Categories Reveal about the Mind. Chicago: University of Chicago Press.

LGwDaF = Langenscheidt e-Großwörterbuch Deutsch als Fremdsprache 5.0.

Lüger, Heinz Helmut (1997): "Anregungen zur Phraseodidaktik". Beiträge zur Fremdsprachenvermittlung 32: 69-120.

Lüger, Heinz-Helmut (2004): "Idiomatische Kompetenz - ein realistisches Lernziel? Thesen zur Phraseodidaktik". In: Lüger, Heinz-Helmut/Rothenhäusler, Rainer (eds.): Linguistik für die Fremdsprache Deutsch. Landau, Empirische Pädagogik: 121-169. (= Beiträge zur Fremdsprachenvermittlung Sonderheft 7).

MacArthur, Fiona/Littlemore, Jeannette (2008): "A discovery approach to figurative language learning with the use of corpora". In: Boers, Frank/Lindstromberg, Seth (eds.): Cognitive Linguistic Approaches to Teaching Vocabulary and Phraseology. Berlin, de Gruyter: 159188. (= Applications of Cognitive Linguistics 6).

Pragglejaz Group (2007): "MIP: A Method for Identifying Metaphorically Used Words in Discourse". Metaphor and Symbol 22.1: 1-39.

Stefanowitsch, Anatol (2006): "Corpus-based approaches to metaphor and metonymy". In: Stefanowitsch, Anatol/Gries, Stefan Th. (eds.): Corpus-Based Approaches to Metaphor and Metonymy. Berlin, de Gruyter: 1-16. (= Trends in Linguistics. Studies and Monographs 171).

Zinken, Jörg (2007): "Discourse metaphors: The link between figurative language and habitual analogies". Cognitive Linguistics 18.3: 445-466.

\section{Anhang}

Redewendungen - Aufgabenblatt (mit der Lösung)

\section{Finden Sie Redewendungen in den folgenden Zeitungstextausschnitten.}

(1) Bereits seit sechs Jahren wohnt Erich (43 Jahre) im Haus der jungen Arbeiter. Ihm gefällt es hier, denn sein Leben verläuft durch das Arbeitsprojekt und die Hilfe von Kaplan Bonetti in geregelten Bahnen. Weihnachten im "Haus" ist für ihn schon zur Routine geworden, und auf den Gottesdienst, das große Fest in der Gemeinschaft und das Geschenkpäckchen freut er sich schon - so wie die anderen auch. (Vorarlberger Nachrichten, 10.12.1998, Feiern und singen um nicht zu weinen)

\section{Redewendung(en): $\quad$ sein Leben verläuft in geregelten Bahnen}

(2) Zwanzig Monate hat der italienische Staatsbürger bereits abgesessen. Der Schreinergeselle

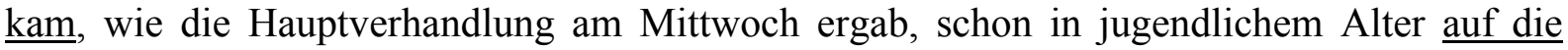
schiefe Bahn. Seinen Lebensunterhalt bestritt er in der Folge mit einer Reihe von Eigentumsdelikten. $1994 \mathrm{kam}$ er in Untersuchungshaft, setzte seine kriminelle Karriere aber schon unmittelbar nach der Entlassung fort. Aus dieser Zeit wurden ihm jetzt von Staatsanwalt Joachim Böhn 25 Einbrüche und Autodiebstähle zur Last gelegt. (Frankfurter Rundschau, 07.03.1997, Fast fünf Jahre Haft wegen Einbruchsserie)

\section{Redewendung(en): auf die schiefe Bahn kommen}

(3) Im Augenblick möchte Mattie eigentlich ihrem Vater für alles die Schuld geben. Sie ist wütend, weil er sie verlassen und ihr Leben dermaßen aus der Bahn geworfen hat. (Berliner Morgenpost, 06.11.1998)

\section{Redewendung(en): aus der Bahn werfen}


Welche inhaltlichen Gemeinsamkeiten können Sie bei den Redewendungen dieser Textausschnitte entdecken?

Alle Redewendungen beschreiben das Leben mit den Komponenten der Bahnfahrt. Das Leben wird in Analogie mit einer Bahnfahrt beschrieben. 